\title{
REVISITING THE FOURIER TRANSFORM ON THE HEISENBERG GROUP
}

\author{
R. LAKShmi Lavanya AND S. ThangaVelu
}

\begin{abstract}
A recent theorem of S. Alesker, S. Artstein-Avidan and V. Milman characterises the Fourier transform on $\mathbb{R}^{n}$ as essentially the only transform on the space of tempered distributions which interchanges convolutions and pointwise products. In this note we study the image of the Schwartz space on the Heisenberg group under the Fourier transform and obtain a similar characterisation for the Fourier transform on the Heisenberg group.
\end{abstract}

2010 Mathematics Subject Classification: 43A30, 43A70, 35K08.

Key words: Heisenberg group, Fourier transform, Fourier-Weyl transform, Schwartz class, heat kernel.

\section{Introduction}

Let $\mathbb{H}^{n}=\mathbb{C}^{n} \times \mathbb{R}$ denote the Heisenberg group equipped with the group law

$$
(z, t)(w, s)=\left(z+w, t+s+\frac{1}{2} \Im(z \cdot \bar{w})\right) .
$$

The group Fourier transform on $\mathbb{H}^{n}$ is defined in terms of the Schrödinger representations $\pi_{\lambda}(z, t)$. Recall that $\pi_{\lambda}(z, t)$ are irreducible unitary representations of $\mathbb{H}^{n}$ realised on the same Hilbert space, namely $L^{2}\left(\mathbb{R}^{n}\right)$; their action is explicitly given by

$$
\pi_{\lambda}(z, t) \varphi(\xi)=e^{i \lambda t} e^{i \lambda\left(x \cdot \xi+\frac{1}{2} x \cdot y\right)} \varphi(\xi+y),
$$

for $\varphi \in L^{2}\left(\mathbb{R}^{n}\right)$. In the above $\lambda \in \mathbb{R}^{*}=\mathbb{R} \backslash\{0\}$ and according to a theorem of Stone and von Neumann any irreducible unitary representation $\rho$ of $\mathbb{H}^{n}$ such that $\rho(0, t)=e^{i \lambda t} I$ is unitarily equivalent to $\pi_{\lambda}$.

The group Fourier transform of a function $f$ on $\mathbb{H}^{n}$, say $f \in L^{1}\left(\mathbb{H}^{n}\right)$, is defined to be the operator valued function

$$
\pi_{\lambda}(f)=\int_{\mathbb{H}^{n}} f(z, t) \pi_{\lambda}(z, t) d z d t .
$$


Note that $\pi_{\lambda}(f)$ is a bounded operator on $L^{2}\left(\mathbb{R}^{n}\right)$ and $\left\|\pi_{\lambda}(f)\right\|_{\text {op }} \leq\|f\|_{1}$. It is well known that when $f \in L^{1} \cap L^{2}\left(\mathbb{H}^{n}\right), \pi_{\lambda}(f)$ is a Hilbert-Schmidt operator and we have the Plancherel theorem

$$
\int_{-\infty}^{\infty}\left\|\pi_{\lambda}(f)\right\|_{H S}^{2} d \mu(\lambda)=\int_{\mathbb{H}^{n}}|f(z, t)|^{2} d z d t,
$$

where $d \mu(\lambda)=c_{n}|\lambda|^{n} d \lambda$ is the Plancherel measure. Let $\mathcal{S}_{2}$ stand for the space of Hilbert-Schmidt operators on $L^{2}\left(\mathbb{R}^{n}\right)$ and define $L^{2}\left(\mathbb{R}^{*}, \mathcal{S}_{2}, d \mu\right)$ to be the space of all functions on $\mathbb{R}^{*}$ taking values in $\mathcal{S}_{2}$, and square integrable with respect to $d \mu(\lambda)$. Then the Plancherel theorem can be restated as: the Fourier transform is a unitary operator from $L^{2}\left(\mathbb{H}^{n}\right)$ onto $L^{2}\left(\mathbb{R}^{*}, \mathcal{S}_{2}, d \mu\right)$. Properties of the group Fourier transform have been studied by several authors starting from the classic work of D. Geller [4].

Apart from the Schrödinger representations $\pi_{\lambda}$, which are all irreducible, we also have certain one dimensional representations on $\mathbb{H}^{n}$. For each $\zeta \in \mathbb{C}^{n}$, these are given by the character $\chi_{\zeta}(z, t)=e^{i \Re z \cdot \bar{\zeta}}$. As we have noted above, the representations $\chi_{\zeta}$ do not occur in the Plancherel theorem as they form a set of zero Plancherel measure. However, we can consider both $\pi_{\lambda}$ and $\chi_{\zeta}$ together and consider the representations

$$
\pi_{\lambda, \zeta}(z, t)=\chi_{\zeta}(z, t) \pi_{\lambda}(z, t)
$$

parametrised by $(\lambda, \zeta) \in \mathbb{R}^{*} \times \mathbb{C}^{n}$. Consequently, we can define the group Fourier transform of a function $f$ on $\mathbb{H}^{n}$ by

$$
\widehat{f}(\lambda, \zeta)=\int_{\mathbb{H}^{n}} f(z, t) \pi_{\lambda, \zeta}(z, t) d z d t .
$$

A simple calculation shows that

$$
\pi_{\lambda, \zeta}(z, t)=\pi_{\lambda}\left(-i \lambda^{-1} \zeta, 0\right) \pi_{\lambda}(z, t) \pi_{\lambda}\left(i \lambda^{-1} \zeta, 0\right)
$$

and hence

$$
\widehat{f}(\lambda, \zeta)=\pi_{\lambda}\left(-i \lambda^{-1} \zeta, 0\right) \pi_{\lambda}(f) \pi_{\lambda}\left(i \lambda^{-1} \zeta, 0\right) .
$$

This operator valued function $\zeta \mapsto \widehat{f}(\lambda, \zeta)$ has been introduced in [9] in connection with a Paley-Wiener theorem for the Heisenberg group. In this paper we will use the above modified Fourier transform, which we call the Fourier-Weyl transform to characterise the image of the Schwartz space under the Fourier transform. Analogous to this, for $S \in L^{2}\left(\mathbb{R}^{*}, \mathcal{S}_{2}, d \mu\right)$, we let

$$
S(\lambda, \zeta)=\pi_{\lambda}\left(-i \lambda^{-1} \zeta, 0\right) S(\lambda) \pi_{\lambda}\left(i \lambda^{-1} \zeta, 0\right) .
$$


As is well known $\pi_{\lambda}(f * g)=\pi_{\lambda}(f) \pi_{\lambda}(g)$, for any $f, g \in L^{1}\left(\mathbb{H}^{n}\right)$. In the Euclidean set up we know that $\mathcal{F}(f * g)(\xi)=(2 \pi)^{\frac{n}{2}} \mathcal{F} f(\xi) \mathcal{F} g(\xi)$ and also $\mathcal{F}(f g)(\xi)=(2 \pi)^{-\frac{n}{2}} \mathcal{F} f * \mathcal{F} g(\xi)$ for $f, g \in L^{1}\left(\mathbb{R}^{n}\right)$. Here $\mathcal{F}$ stands for the Euclidean Fourier transform. Moreover, $\mathcal{F}$ takes the Schwartz space $\mathcal{S}\left(\mathbb{R}^{n}\right)$ onto itself; also it takes $\mathcal{S}^{\prime}\left(\mathbb{R}^{n}\right)$, the space of tempered distributions onto itself. It has been shown by S. Alesker, S. ArtsteinAvidan and V. Milman that these properties completely characterise the Euclidean Fourier transform.

Theorem 1.1 (S. Alesker, S. Artstein-Avidan and V. Milman [1]). Assume we are given a transform $T: \mathcal{S}\left(\mathbb{R}^{n}\right) \rightarrow \mathcal{S}\left(\mathbb{R}^{n}\right)$ that admits a bijective extension $T^{\prime}: \mathcal{S}^{\prime}\left(\mathbb{R}^{n}\right) \rightarrow \mathcal{S}^{\prime}\left(\mathbb{R}^{n}\right)$ such that

(i) for every $f \in \mathcal{S}\left(\mathbb{R}^{n}\right)$ and $\varphi \in \mathcal{S}^{\prime}\left(\mathbb{R}^{n}\right), T^{\prime}(f * \varphi)=T(f) T^{\prime}(\varphi)$,

(ii) for every $f \in \mathcal{S}\left(\mathbb{R}^{n}\right)$ and $\varphi \in \mathcal{S}^{\prime}\left(\mathbb{R}^{n}\right)$, we have $T^{\prime}(f \varphi)=T(f) *$ $T^{\prime}(\varphi)$.

Then, $T$ is essentially the Fourier transform $\mathcal{F}$ : that is, for some $B \in$ $G L_{n}(\mathbb{R})$ with $|\operatorname{det} B|=1$, we have either $T f=\mathcal{F}(f \circ B)$ or $T f=$ $\overline{\mathcal{F}(f \circ B)}$, for all $f$ in $\mathcal{S}\left(\mathbb{R}^{n}\right)$.

In an earlier paper [7], we obtained some characterisations of the Weyl transform and the Heisenberg group Fourier transform assuming the map to be a continuous linear operator satisfying certain conditions. In this paper, we obtain another characterisation, analogous to Theorem 1.1, for the Heisenberg group Fourier transform where we do not assume the map to be linear or continuous, but we only assume it to be a bijection.

Let $\mathcal{S}\left(\mathbb{H}^{n}\right)$ stand for the Schwartz space on $\mathbb{H}^{n}$. Denote by $\widehat{\mathcal{S}}\left(\mathbb{H}^{n}\right)$ the image of $\mathcal{S}\left(\mathbb{H}^{n}\right)$ under the group Fourier transform $f \rightarrow \pi_{\lambda}(f)$. It is well known that the Fourier transform is an isometry from $L^{2}\left(\mathbb{H}^{n}\right)$ onto $L^{2}\left(\mathbb{R}^{*}, \mathcal{S}_{2}, d \mu\right)$. In view of this $\widehat{\mathcal{S}}\left(\mathbb{H}^{n}\right)$ is a subspace of $L^{2}\left(\mathbb{R}^{*}, \mathcal{S}_{2}, d \mu\right)$. In Section 2, we give a characterisation of $\widehat{\mathcal{S}}\left(\mathbb{H}^{n}\right)$.

For functions $f, g \in L^{1}\left(\mathbb{H}^{n}\right)$ their convolution defined by

$$
(f * g)(z, t)=\int_{\mathbb{H}^{n}} f((z, t)(-w,-s)) g(w, s) d w d s
$$

is also integrable on $\mathbb{H}^{n}$ and satisfies

$$
\pi_{\lambda}(f * g)=\pi_{\lambda}(f) \pi_{\lambda}(g) .
$$


Given $f$ and $g$ from $L^{1}\left(\mathbb{H}^{n}\right)$ we denote by $f *_{3} g$ the convolution in the central variable; thus

$$
f *_{3} g(z, t)=\int_{-\infty}^{\infty} f(z, t-s) g(z, s) d s .
$$

We will show below that the Fourier-Weyl transform satisfies

$$
\left(f *_{3} g\right)^{\wedge}(\lambda, \zeta)=2 \pi \int_{\mathbb{C}^{n}} \mathcal{F}^{-1} f(w-\zeta, \lambda) \widehat{g}(\lambda, w) d w,
$$

where $\mathcal{F}^{-1}$ is the inverse Euclidean Fourier transform on $\mathbb{C}^{n} \times \mathbb{R}$. We consider the above as the analogue of

$$
\mathcal{F}(f g)(\xi)=(2 \pi)^{-\frac{n}{2}}(\mathcal{F} f * \mathcal{F} g)(\xi)
$$

for the Euclidean Fourier transform. If we denote by $R_{(z, t)}$, the right translation on $\mathbb{H}^{n}$, which gives rise to a translation on functions on $\mathbb{H}^{n}$ given by $\left(R_{(z, t)} f\right)(w, s)=f((w, s)(z, t))$, then the Fourier transform satisfies

$$
\pi_{\lambda}\left(R_{(z, t)} f\right)=\pi_{\lambda}(f) \pi_{\lambda}(z, t)^{*} .
$$

We show that the properties (1.1), (1.2) and (1.3) characterise the Fourier transform on $\mathbb{H}^{n}$. The Heisenberg group is equipped with the non-isotropic dilation $\delta_{r}(z, t)=\left(r z, r^{2} t\right)$ with respect to which we have

$$
\pi_{\lambda}\left(\delta_{r} f\right)=r^{-(2 n+2)} d_{r} \circ \pi_{\frac{\lambda}{r^{2}}}(f) \circ d_{r}^{-1},
$$

where $d_{r} \varphi(\xi)=\varphi(r \xi)$ is the standard dilation. In the next theorem we let $\operatorname{Tg}(\lambda, w)$ stand for $\pi_{\lambda}\left(-i \lambda^{-1} w, 0\right) T g(\lambda) \pi_{\lambda}\left(i \lambda^{-1} w, 0\right)$.

Theorem 1.2. Let $T: \mathcal{S}\left(\mathbb{H}^{n}\right) \rightarrow \widehat{\mathcal{S}}\left(\mathbb{H}^{n}\right)$ be a bijection which satisfies

(i) $T(f * g)=T f T g$, and

(ii) $T\left(f *_{3} g\right)(\lambda)=2 \pi \int_{\mathbb{C}^{n}} \mathcal{F}^{-1} f(w, \lambda) T g(\lambda, w) d w$.

Then there exists a map $\zeta: \mathbb{R}^{*} \rightarrow \mathbb{C}^{n}$ such that $\operatorname{T} f(\lambda)=\widehat{f}(\lambda, \zeta(\lambda))$.

Remark 1.1. The above theorem is the analogue of Theorem 1.1 for the group Fourier transform on $\mathbb{H}^{n}$. A similar result for the Weyl transform was proved in $[6]$. Note that we do not assume anything on the action of the transform on the space of tempered distributions. The proof of the above theorem indicates that similar assumptions on the space of tempered distributions are not necessary in the characterisation of the Weyl transform obtained in $[6]$. 
Corollary 1.3. In addition to the hypotheses of Theorem 1.2, if the map $T$ satisfies

$$
T\left(R_{(z, 0)} f\right)(\lambda)=T f(\lambda) \pi_{\lambda}(z)^{*},
$$

then $\operatorname{Tf}(\lambda)=\pi_{\lambda}(f)$.

Corollary 1.4. If $T$ is as in Theorem 1.2 and $T$ satisfies

$$
T\left(\delta_{r} f\right)(\lambda)=r^{-(2 n+2)} d_{r} \circ T f\left(\frac{\lambda}{r^{2}}\right) \circ d_{r}^{-1}
$$

then we get

for some $\zeta_{0}, \zeta_{1} \in \mathbb{C}^{n}$.

$$
T f(\lambda)= \begin{cases}\widehat{f}\left(\lambda, \sqrt{\lambda} \zeta_{0}\right), & \lambda>0 \\ \widehat{f}\left(\lambda, \sqrt{|\lambda|} \zeta_{1}\right), & \lambda<0,\end{cases}
$$

As mentioned above we obtain a characterisation of $\widehat{\mathcal{S}}\left(\mathbb{H}^{n}\right)$. As the results are technical we do not state them here. In the last section we consider the subspace of $L^{2}\left(\mathbb{H}^{n}\right)$ consisting of functions of the form $f *_{3} q_{s}$ where $q_{s}$ is the heat kernel on $\mathbb{H}^{n}$ associated to the sublaplacian.

By slightly changing the notation we write the Fourier-Weyl transform of a function $g$ as

$$
\widetilde{g}(\lambda, x, u)=\widehat{g}(\lambda, x+i u), \quad x, u \in \mathbb{R}^{n} .
$$

With this notation we address the question of characterising functions of the form $g=f *_{3} q_{s}$ for a fixed $s>0$ under the Fourier-Weyl transform. We prove the following result. For $\lambda \in \mathbb{R}^{*}$ and $s>0$ we let

$$
\omega_{s, \lambda}(y, v)=c_{n} \lambda^{-n}(\sinh 2 \lambda s)^{n} e^{-\frac{2}{\lambda}(\tanh \lambda s)\left(|y|^{2}+|v|^{2}\right)},
$$

where $y, v \in \mathbb{R}^{n}$.

Theorem 1.5. A function $g \in L^{2}\left(\mathbb{H}^{n}\right)$ can be written as $g=f *_{3} q_{s}$ for some $f \in L^{2}\left(\mathbb{H}^{n}\right)$ if and only if for each $\lambda, \widetilde{g}(\lambda, x, u)$ extends to $\mathbb{C}^{n} \times \mathbb{C}^{n}$ as an operator valued entire function $\widetilde{g}(\lambda, z, w)$ with $z=x+i y$ and $w=u+i v$ which satisfies

$$
\begin{aligned}
\int_{-\infty}^{\infty} \int_{\mathbb{C}^{2 n}}\|\widetilde{g}(\lambda, z, w) \varphi\|_{2}^{2} \omega_{s, \lambda}(y, v) & d z d w d \lambda \\
& =c_{n, s} \int_{\mathbb{H}^{n}}|f(z, t)|^{2} d z d t \int_{\mathbb{R}^{n}}|\varphi(\xi)|^{2} d \xi
\end{aligned}
$$

for every $\varphi \in L^{2}\left(\mathbb{R}^{n}\right)$.

We prove this theorem in Section 4. 


\section{Properties of the Fourier transform}

In this section we recall some properties of the group Fourier transform on $\mathbb{H}^{n}$ most of which are well known. We also establish the property (1.2) for the Fourier transform. Let $\mathbb{H}^{n}$ be the $(2 n+1)$-dimensional Heisenberg group with the underlying manifold $\mathbb{C}^{n} \times \mathbb{R}$, and the group law given by

$$
(z, t)(w, s)=\left(z+w, t+s+\frac{1}{2} \Im(z \cdot \bar{w})\right), \quad(z, t),(w, s) \in \mathbb{H}^{n} .
$$

The Haar measure on $\mathbb{H}^{n}$ is the Lebesgue measure $d z d t$ on $\mathbb{C}^{n} \times \mathbb{R}$. The Stone-von Neumann theorem states that all the infinite-dimensional irreducible unitary representations of $\mathbb{H}^{n}$, acting on $L^{2}\left(\mathbb{R}^{n}\right)$, are parametrised by $\lambda \in \mathbb{R}^{*}$, and are given by

$$
\pi_{\lambda}(z, t) \varphi(\xi)=e^{i \lambda t} e^{i \lambda\left(x \cdot \xi+\frac{1}{2} x \cdot y\right)} \varphi(\xi+y),
$$

for $\varphi \in L^{2}\left(\mathbb{R}^{n}\right), \xi \in \mathbb{R}^{n}$ and $(z, t)=(x, y, t) \in \mathbb{H}^{n}$. We write $\pi_{\lambda}(z)=$ $\pi_{\lambda}(z, 0)$ so that $\pi_{\lambda}(z, t)=e^{i \lambda t} \pi_{\lambda}(z)$.

For $\lambda \in \mathbb{R}^{*}$ and $g \in L^{1}\left(\mathbb{C}^{n}\right)$, consider the operator

$$
W_{\lambda}(g)=\int_{\mathbb{C}^{n}} g(z) \pi_{\lambda}(z) d z .
$$

When $\lambda=1$, we call this the Weyl transform of $g$. For functions $f, g \in$ $L^{1}\left(\mathbb{C}^{n}\right)$, their $\lambda$-twisted convolution is defined as

$$
f *_{\lambda} g(z)=\int_{\mathbb{C}^{n}} f(z-w) g(w) e^{i \frac{\lambda}{2} \Im(z \cdot \bar{w})} d w .
$$

The operators $W_{\lambda}$ are continuous, linear and map $L^{1}\left(\mathbb{C}^{n}\right)$ into $\mathcal{B}\left(L^{2}\left(\mathbb{R}^{n}\right)\right)$. Moreover, $W_{\lambda}\left(f *_{\lambda} g\right)=W_{\lambda}(f) W_{\lambda}(g), f, g \in L^{1}\left(\mathbb{C}^{n}\right)$.

For an integrable function $f$ on $\mathbb{H}^{n}$, its Fourier transform is the operator-valued function defined by

$$
\pi_{\lambda}(f) \varphi=\int_{\mathbb{H}^{n}} f(z, t) \pi_{\lambda}(z, t) \varphi d z d t, \quad \varphi \in L^{2}\left(\mathbb{R}^{n}\right), \quad \lambda \in \mathbb{R}^{*} .
$$

The Fourier transform is a bounded operator on $L^{2}\left(\mathbb{R}^{n}\right)$ and it satisfies $\left\|\pi_{\lambda}(f)\right\|_{\text {op }} \leq\|f\|_{1}$. In particular, when $f$ is also in $L^{2}\left(\mathbb{H}^{n}\right), \pi_{\lambda}(f)$ is a Hilbert-Schmidt operator. 
The convolution of functions $f, g \in L^{1}\left(\mathbb{H}^{n}\right)$ is defined as

$$
f * g(z, t)=\int_{\mathbb{H}^{n}} f((z, t)(-w,-s)) g(w, s) d w d s .
$$

The Fourier transform satisfies $\pi_{\lambda}(f * g)=\pi_{\lambda}(f) \pi_{\lambda}(g), f, g \in L^{1}\left(\mathbb{H}^{n}\right)$.

Let $\mathcal{S}_{2}$ denote the space of Hilbert-Schmidt operators on $L^{2}\left(\mathbb{R}^{n}\right)$. Then $\mathcal{S}_{2}$ is a Hilbert space with the inner product $(T, S)=\operatorname{tr}\left(T S^{*}\right)$. Let $\|S\|_{H S}$ denote the Hilbert-Schmidt norm of an operator $S \in \mathcal{S}_{2}$. Let $d \mu(\lambda)=(2 \pi)^{-n-1}|\lambda|^{n} d \lambda$ and let $L^{2}\left(\mathbb{R}^{*}, \mathcal{S}_{2}, d \mu\right)$ denote the space of functions on $\mathbb{R}^{*}$ taking values in $\mathcal{S}_{2}$ and square integrable with respect to the measure $d \mu$.

The following well-known result summarises an important property of the Fourier transform, see [10].

Theorem 2.1. The group Fourier transform is an isometric isomorphism between $L^{2}\left(\mathbb{H}^{n}\right)$ and $L^{2}\left(\mathbb{R}^{*}, \mathcal{S}_{2}, d \mu\right)$.

We denote by $\mathcal{F}^{-1} f(z, \lambda)$ the inverse Euclidean Fourier transform of the function $f$ on $\mathbb{C}^{n} \times \mathbb{R}$ :

$$
\mathcal{F}^{-1} f(z, \lambda)=(2 \pi)^{-n-\frac{1}{2}} \int_{\mathbb{C}^{n} \times \mathbb{R}} e^{i(\Re(z \cdot \bar{w})+\lambda s)} f(w, s) d w d s .
$$

For $\lambda \in \mathbb{R}$, the partial inverse Fourier transform of $f$ in the third variable, denoted by $f^{\lambda}$, is defined as

$$
f^{\lambda}(w)=\int_{\mathbb{R}} f(w, t) e^{i \lambda t} d t .
$$

Let $d_{r}$ be the standard dilation on $\mathbb{R}^{n}$ and $\delta_{r}$ the non-isotropic dilation on $\mathbb{H}^{n}$ defined by $\delta_{r}(z, t)=\left(r z, r^{2} t\right)$. We also let $\left(\delta_{r} f\right)(z, t)=f\left(\delta_{r}(z, t)\right)$. An easy calculation shows that $\pi_{\lambda}(r z)=d_{r}^{-1} \circ \pi_{\lambda r^{2}}(z) \circ d_{r}$. Consequently,

$$
\begin{aligned}
\pi_{\lambda}\left(\delta_{r} f\right) & =\int_{\mathbb{H}^{n}} f\left(r z, r^{2} t\right) \pi_{\lambda}(z, t) d z d t \\
& =r^{-2 n-2} \int_{\mathbb{C}^{n}} f^{\lambda / r^{2}}(z) \pi_{\lambda / r^{2}}\left(r^{-1} z\right) d z .
\end{aligned}
$$

In view of the above formula for $\pi_{\lambda}(r z)$ we get

$$
\pi_{\lambda}\left(\delta_{r} f\right)=r^{-2 n-2} d_{r} \circ \pi_{\frac{\lambda}{r^{2}}}(f) \circ d_{r}^{-1} .
$$

We also have

$$
\left(\delta_{r} f\right)^{\wedge}(\lambda, \zeta)=r^{-2 n-2} d_{r} \circ \widehat{f}\left(\frac{\lambda}{r^{2}}, r^{-1} \zeta\right) \circ d_{r}^{-1} .
$$



form.

We can now prove the following theorem for the Fourier-Weyl trans-

Theorem 2.2. For $f, g \in \mathcal{S}\left(\mathbb{H}^{n}\right)$ and $\zeta \in \mathbb{C}^{n}$

$$
\left(f *_{3} g\right)^{\wedge}(\lambda, \zeta)=2 \pi \int_{\mathbb{C}^{n}} \mathcal{F}^{-1} f(w-\zeta, \lambda) \widehat{g}(\lambda, w) d w .
$$

Proof: Let $\varphi, \psi \in L^{2}\left(\mathbb{R}^{n}\right)$. Then by the definition of $\widehat{g}(\lambda, w)$ we have

$$
(\widehat{g}(\lambda, z) \varphi, \psi)=\int_{\mathbb{C}^{n}} g^{\lambda}(w) e^{-i \Re z \cdot \bar{w}}\left(\pi_{\lambda}(w) \varphi, \psi\right) d w .
$$

Since $\left(f *_{3} g\right)^{\lambda}(z)=(2 \pi)^{\frac{1}{2}} f^{\lambda}(z) g^{\lambda}(z)$ we have

$$
\left(\left(f *_{3} g\right)^{\wedge}(\lambda, \zeta) \varphi, \psi\right)=(2 \pi)^{\frac{1}{2}} \int_{\mathbb{C}^{n}} f^{\lambda}(w) g^{\lambda}(w)\left(\pi_{\lambda}(w) \varphi, \psi\right) e^{-i \Re \zeta \cdot \bar{w}} d w .
$$

The theorem follows as the Euclidean Fourier transform converts products into convolutions.

In order to study the image of $\mathcal{S}\left(\mathbb{H}^{n}\right)$ under the Fourier transform we need to introduce the following operators (see [4]). Let $A$ be a (possibly unbounded) densely defined operator. Then for any bounded operator $S$ on $L^{2}\left(\mathbb{R}^{n}\right)$ which maps $\operatorname{dom}(A)$ into itself we define the derivation

$$
\partial_{A} S=[S, A]=S A-A S .
$$

For $j=1,2, \ldots, n$, let $Q_{j} \varphi(\xi)=\xi_{j} \varphi(\xi)$ and $P_{j} \varphi(\xi)=\frac{\partial}{\partial \xi_{j}} \varphi(\xi)$ for a function $\varphi$ on $\mathbb{R}^{n}$. We let $\partial_{P_{j}}$ and $\partial_{Q_{j}}$ stand for the corresponding derivations. For $j=1,2, \ldots, n$, we also define

$$
M_{P_{j}} S=S P_{j}+P_{j} S, \quad M_{Q_{j}} S=S Q_{j}+Q_{j} S .
$$

On the Heisenberg group we have the following left invariant vector fields:

$$
X_{j}=\frac{\partial}{\partial x_{j}}+\frac{1}{2} y_{j} \frac{\partial}{\partial t}, \quad Y_{j}=\frac{\partial}{\partial y_{j}}-\frac{1}{2} x_{j} \frac{\partial}{\partial t}, \quad j=1,2, \ldots, n .
$$

We let $\widetilde{X}_{j}$ and $\widetilde{Y}_{j}$ stand for the corresponding right invariant vector fields:

$$
\widetilde{X}_{j}=\frac{\partial}{\partial x_{j}}-\frac{1}{2} y_{j} \frac{\partial}{\partial t}, \quad \widetilde{Y}_{j}=\frac{\partial}{\partial y_{j}}+\frac{1}{2} x_{j} \frac{\partial}{\partial t}, \quad j=1,2, \ldots, n .
$$

The following proposition is implicit in the work of Geller [4]. 
Proposition 2.3. For $f \in \mathcal{S}\left(\mathbb{H}^{n}\right)$ and $\lambda \in \mathbb{R}^{*}$, we have, for $j=$ $1,2, \ldots, n$,

(i) $\pi_{\lambda}\left(-i x_{j} f\right)=\lambda^{-1} \partial_{P_{j}} \pi_{\lambda}(f)$,

(ii) $\pi_{\lambda}\left(y_{j} f\right)=\partial_{Q_{j}} \pi_{\lambda}(f)$,

(iii) $\pi_{\lambda}\left(i X_{j} f\right)=\lambda \pi_{\lambda}(f) Q_{j}$,

(iv) $\pi_{\lambda}\left(-Y_{j} f\right)=\pi_{\lambda}(f) P_{j}$,

(v) $\pi_{\lambda}\left(i \tilde{X}_{j} f\right)=\lambda Q_{j} \pi_{\lambda}(f)$,

(vi) $\pi_{\lambda}\left(-\widetilde{Y}_{j} f\right)=P_{j} \pi_{\lambda}(f)$.

We would also like to express $\pi_{\lambda}(i t f)$ in terms of $\pi_{\lambda}(f)$. Let $\Lambda$ be the operator defined by

$$
\Lambda \pi_{\lambda}(f)=\frac{\partial}{\partial \lambda} \pi_{\lambda}(f)+\frac{1}{\lambda} \sum_{j=1}^{n} \pi_{\lambda}\left(\frac{\partial}{\partial x_{j}}\left(x_{j} f\right)\right),
$$

where $x=\Re z \in \mathbb{R}^{n}$. Then we have

Proposition 2.4. For $f \in \mathcal{S}\left(\mathbb{H}^{n}\right)$ and $\lambda \in \mathbb{R}^{*}$,

$$
\pi_{\lambda}(i t f)=\Lambda \pi_{\lambda}(f) .
$$

We refer to Geller [4] for a proof. We remark that Propositions 2.3 and 2.4 are easily proved starting with the equation

$$
\left(\pi_{\lambda}(f) \varphi, \psi\right)=\int_{\mathbb{C}^{n}} f^{\lambda}(z)\left(\pi_{\lambda}(z) \varphi, \psi\right) d z .
$$

We leave the details to the reader. For multi-indices $\alpha, \beta, \mu, \nu \in \mathbb{N}^{n}$ we define $\partial_{P}^{\alpha}, \partial_{Q}^{\beta}, M_{Q}^{\mu}$ and $M_{P}^{\nu}$ in the usual way, e.g. $\partial_{P}^{\alpha}=\partial_{P_{1}}^{\alpha_{1}} \partial_{P_{2}}^{\alpha_{2}} \ldots \partial_{P_{n}}^{\alpha_{n}}$, etc. Let $S(\lambda)$ be an operator valued function on $\mathbb{R}^{*}$. For each $N \in \mathbb{N}$ we define a seminorm

$$
\|S\|_{N}^{2}=\sup \int_{-\infty}^{\infty}\left\|\left(\lambda^{-1} \partial_{P}\right)^{\mu} \partial_{Q}^{\nu} \Lambda^{k}\left(\lambda M_{Q}\right)^{\alpha} M_{P}^{\beta} \lambda^{j} S(\lambda)\right\|_{H S}^{2} d \mu(\lambda),
$$

where the supremum is taken over all multi-indices $\mu, \nu, \alpha, \beta \in \mathbb{N}^{n}$ and $j, k \in \mathbb{N}$ such that $|\mu|+|\nu|+|\alpha|+|\beta|+j+k \leq N$. Let $\mathcal{S}\left(\mathbb{R}^{*}, \mathcal{S}_{2}\right)$ stand for the set of all operator valued functions $S$ on $\mathbb{R}^{*}$ for which the seminorms $\|S\|_{N}$ are finite for all $N \in \mathbb{N}$. We can make $\mathcal{S}\left(\mathbb{R}^{*}, \mathcal{S}_{2}\right)$ into a topological vector space using these seminorms.

Theorem 2.5. The Fourier transform $f \rightarrow \pi_{\lambda}(f)$ is a topological isomorphism from $\mathcal{S}\left(\mathbb{H}^{n}\right)$ onto $\mathcal{S}\left(\mathbb{R}^{*}, \mathcal{S}_{2}\right)$. 
Proof: Let $f \in \mathcal{S}\left(\mathbb{H}^{n}\right)$ and $\alpha, \beta, \mu, \nu \in \mathbb{N}^{n}, j, k \in \mathbb{N}$. Then the function

$$
f_{\alpha \beta \mu \nu j k}(x, y, t)=(-i x)^{\mu} y^{\nu}(i t)^{k}\left(i \partial_{x}\right)^{\alpha}\left(-\partial_{y}\right)^{\beta}\left(-i \partial_{t}\right)^{j} f(x, y, t)
$$

belongs to $L^{2}\left(\mathbb{H}^{n}\right)$. As $\frac{\partial}{\partial x_{j}}=\frac{1}{2}\left(X_{j}+\widetilde{X}_{j}\right)$ we note that $\pi_{\lambda}\left(\left(i \partial_{x}\right)^{\alpha} f\right)=$ $\left(\lambda M_{Q}\right)^{\alpha} \pi_{\lambda}(f)$. Similarly $\pi_{\lambda}\left(\left(-\partial_{y}\right)^{\beta} f\right)=M_{P}^{\beta} \pi_{\lambda}(f)$. Therefore, in view of Propositions 2.3 and 2.4,

$$
\pi_{\lambda}\left(f_{\alpha \beta \mu \nu j k}\right)=\left(\lambda^{-1} \partial_{P}\right)^{\mu} \partial_{Q}^{\nu} \Lambda^{k}\left(\lambda M_{Q}\right)^{\alpha} M_{P}^{\beta} \lambda^{j} \pi_{\lambda}(f)
$$

is a Hilbert-Schmidt operator and

$$
\int_{\mathbb{R}^{*}}\left\|\pi_{\lambda}\left(f_{\alpha \beta \mu \nu j k}\right)\right\|_{H S}^{2} d \mu(\lambda)=c \int_{\mathbb{H}^{n}}\left|f_{\alpha \beta \mu \nu j k}(z, t)\right|^{2} d z d t .
$$

Thus the group Fourier transform takes $\mathcal{S}\left(\mathbb{H}^{n}\right)$ into $\mathcal{S}\left(\mathbb{R}^{*}, \mathcal{S}_{2}\right)$. Also note that there is a one to one correspondence between seminorms defining the respective topologies on $\mathcal{S}\left(\mathbb{H}^{n}\right)$ and $\mathcal{S}\left(\mathbb{R}^{*}, \mathcal{S}_{2}\right)$.

To prove the surjectivity, suppose $S \in \mathcal{S}\left(\mathbb{R}^{*}, \mathcal{S}_{2}\right)$. Then we get $S(\lambda)=$ $\pi_{\lambda}(f)$ for $f \in L^{2}\left(\mathbb{H}^{n}\right)$. The fact that

$$
\int_{-\infty}^{\infty}\left\|\left(\lambda^{-1} \partial_{P}\right)^{\mu} \partial_{Q}^{\nu} \Lambda^{k}\left(\lambda M_{Q}\right)^{\alpha} M_{P}^{\beta} \lambda^{j} S(\lambda)\right\|_{H S}^{2} d \mu(\lambda)<\infty
$$

translates into

$$
\int_{\mathbb{H}^{n}}\left|f_{\alpha \beta \mu \nu j k}(z, t)\right|^{2} d z d t<\infty .
$$

Consequently, we infer that $f \in \mathcal{S}\left(\mathbb{H}^{n}\right)$, which proves the surjectivity.

The above theorem states that $\widehat{\mathcal{S}}\left(\mathbb{H}^{n}\right)=\mathcal{S}\left(\mathbb{R}^{*}, \mathcal{S}_{2}\right)$.

\section{A characterisation of the Fourier transform}

In this section we prove Theorem 1.2 stated in the introduction. If $f \in \mathcal{S}\left(\mathbb{H}^{n}\right)$, then $T f \in \widehat{\mathcal{S}}\left(\mathbb{H}^{n}\right)$ and hence we can find $g \in \mathcal{S}\left(\mathbb{H}^{n}\right)$ so that $T f(\lambda)=\pi_{\lambda}(g)$. Defining $U f=g$, where $g$ is as above, we get a bijection of $\mathcal{S}\left(\mathbb{H}^{n}\right)$ onto itself such that $\operatorname{Tf}(\lambda)=\pi_{\lambda}(U f)$ for all $f \in \mathcal{S}\left(\mathbb{H}^{n}\right)$. For $f, g \in \mathcal{S}\left(\mathbb{H}^{n}\right)$, the assumption (i) of Theorem 1.2 gives

$\pi_{\lambda}(U(f * g))=T(f * g)(\lambda)=T f(\lambda) T g(\lambda)=\pi_{\lambda}(U f) \pi_{\lambda}(U g)=\pi_{\lambda}(U f * U g)$.

This implies that

$$
U(f * g)=U f * U g, \quad f, g \in \mathcal{S}\left(\mathbb{H}^{n}\right) .
$$


By the assumption (ii),

$$
\begin{aligned}
T\left(f *_{3} g\right)(\lambda) & =2 \pi \int_{\mathbb{C}^{n}} \mathcal{F}^{-1} f(w, \lambda) T g(\lambda, w) d w \\
& =2 \pi \int_{\mathbb{C}^{n}} \mathcal{F}^{-1} f(w, \lambda)(U g)^{\wedge}(\lambda, w) d w,
\end{aligned}
$$

which, combined with Theorem 2.2, gives

$$
\pi_{\lambda}\left(U\left(f *_{3} g\right)\right)=\pi_{\lambda}\left(f *_{3} U g\right) .
$$

As $f *_{3} g=g *_{3} f$ we obtain

$$
U\left(f *_{3} g\right)=f *_{3} U g=g *_{3} U f,
$$

and consequently

$$
f^{\lambda}(z)(U g)^{\lambda}(z)=g^{\lambda}(z)(U f)^{\lambda}(z)
$$

for every $\lambda \in \mathbb{R}^{*}$.

For a given $\lambda \in \mathbb{R}^{*}$ let $f \in \mathcal{S}\left(\mathbb{H}^{n}\right)$ be such that $f^{\lambda}(z)$ vanishes nowhere on $\mathbb{C}^{n}$. Then for any $g \in \mathcal{S}\left(\mathbb{H}^{n}\right)$

$$
(U g)^{\lambda}(z)=\frac{(U f)^{\lambda}(z)}{f^{\lambda}(z)} g^{\lambda}(z)=\varphi_{\lambda}(z) g^{\lambda}(z) .
$$

The function $\varphi_{\lambda}(z)$ is continuous by definition. For $g, h \in \mathcal{S}\left(\mathbb{H}^{n}\right)$

$$
\begin{aligned}
\varphi_{\lambda}(z)(g * h)^{\lambda}(z) & =(U(g * h))^{\lambda}(z)=(U g * U h)^{\lambda}(z) \\
& =\left(\varphi_{\lambda} g^{\lambda}\right) *_{\lambda}\left(\varphi_{\lambda} h^{\lambda}\right)(z) .
\end{aligned}
$$

Therefore

$$
\int_{\mathbb{C}^{n}}\left(\varphi_{\lambda}(z)-\varphi_{\lambda}(z-w) \varphi_{\lambda}(w)\right) g^{\lambda}(z-w) h^{\lambda}(w) e^{i \frac{\lambda}{2} \Im(z \cdot \bar{w})} d w=0 .
$$

By taking $h(w, s)=e^{-\frac{1}{2}\left(s^{2}+t|w|^{2}\right)}$ and $g \in \mathcal{S}\left(\mathbb{H}^{n}\right)$ for which $g^{\lambda}$ is compactly supported and letting $t \rightarrow 0$ we get

$$
\int_{\mathbb{C}^{n}}\left(\varphi_{\lambda}(z)-\varphi_{\lambda}(z-w) \varphi_{\lambda}(w)\right) g^{\lambda}(z-w) e^{i \frac{\lambda}{2} \Im(z \cdot \bar{w})} d w=0 .
$$

As this is true for all such $g$ we get

$$
\varphi_{\lambda}(z)=\varphi_{\lambda}(z-w) \varphi_{\lambda}(w)
$$

for a.e. $w \in \mathbb{C}^{n}$. The equation holds true for all $w \in \mathbb{C}^{n}$ as $\varphi_{\lambda}$ is continuous. Thus we get

$$
\varphi_{\lambda}(z+w)=\varphi_{\lambda}(z) \varphi_{\lambda}(w)
$$


Consequently, $\varphi_{\lambda}(0)=\varphi_{\lambda}(0)^{2}$ which means $\varphi_{\lambda}(0)=0$ or $\varphi_{\lambda}(0)=1$. The case $\varphi_{\lambda}(0)=0$ is ruled out as it leads to $\varphi_{\lambda}(z)=0$ for all $z$ and hence $(U g)^{\lambda}(z)=\varphi_{\lambda}(z) g^{\lambda}(z)=0$ so that $U=0$. With $\varphi_{\lambda}(0)=1$ and the fact that $U$ takes $\mathcal{S}\left(\mathbb{H}^{n}\right)$ onto $\mathcal{S}\left(\mathbb{H}^{n}\right)$ we obtain $\varphi_{\lambda}(z)=e^{-i \Re(z \cdot \overline{\zeta(\lambda)})}$ for some $\zeta(\lambda) \in \mathbb{C}^{n}$. Therefore,

$$
T f(\lambda)=\pi_{\lambda}(U f)=\int_{\mathbb{C}^{n}} e^{-i \Re(z \cdot \overline{\zeta(\lambda)})} f^{\lambda}(z) \pi_{\lambda}(z) d z .
$$

Thus $T f(\lambda)=\widehat{f}(\lambda, \zeta(\lambda)), f \in \mathcal{S}\left(\mathbb{H}^{n}\right)$.

Proof of Corollary 1.3: By (1.4) we have $T\left(R_{(z, 0)} f\right)(\lambda)=T f(\lambda) \pi_{\lambda}(z)^{*}$. Since $T f(\lambda)=\widehat{f}(\lambda, \zeta(\lambda))$ this gives

$$
\begin{aligned}
T f(\lambda) \pi_{\lambda}(z)^{*} & =\left(R_{(z, 0)} f\right)^{\wedge}(\lambda, \zeta(\lambda)) \\
\text { i.e., } \quad \widehat{f}(\lambda, \zeta(\lambda)) \pi_{\lambda}(z)^{*} & =\pi_{\lambda}\left(-i \lambda^{-1} \zeta(\lambda)\right) \pi_{\lambda}(f) \pi_{\lambda}(z)^{*} \pi_{\lambda}\left(i \lambda^{-1} \zeta(\lambda)\right)
\end{aligned}
$$

for any $z \in \mathbb{C}^{n}$ and $f \in \mathcal{S}\left(\mathbb{H}^{n}\right)$. This implies

$$
\begin{aligned}
\pi_{\lambda}\left(i \lambda^{-1} \zeta(\lambda)\right) \pi_{\lambda}(z)^{*} & =\pi_{\lambda}(z)^{*} \pi_{\lambda}\left(i \lambda^{-1} \zeta(\lambda)\right), & \\
\text { i.e., } \quad e^{i \Re(z \cdot \overline{\zeta(\lambda)})} \pi_{\lambda}(z)^{*} & =\pi_{\lambda}(z)^{*}, & \text { for all } z \in \mathbb{C}^{n} .
\end{aligned}
$$

This forces $\zeta(\lambda)=0$ and hence $T f(\lambda)=\pi_{\lambda}(f)$ as desired.

We are left with deducing Corollary 1.4.

Proof of Corollary 1.4: On the one hand by (2.1),

$$
T\left(\delta_{r} f\right)(\lambda)=\left(\delta_{r} f\right)^{\wedge}(\lambda, \zeta(\lambda))=r^{-(2 n+2)} d_{r} \circ \widehat{f}\left(\frac{\lambda}{r^{2}}, r^{-1} \zeta(\lambda)\right) \circ d_{r}^{-1} .
$$

On the other hand by (1.5),

$T\left(\delta_{r} f\right)(\lambda)=r^{-(2 n+2)} d_{r} \circ T f\left(\frac{\lambda}{r^{2}}\right) \circ d_{r}^{-1}=r^{-(2 n+2)} d_{r} \circ \widehat{f}\left(\frac{\lambda}{r^{2}}, \zeta\left(\frac{\lambda}{r^{2}}\right)\right) \circ d_{r}^{-1}$.

Therefore, we get $r^{-1} \zeta\left(\lambda r^{2}\right)=\zeta(\lambda)$ for all $r>0$. This leads to $\zeta(\lambda)=$ $\sqrt{|\lambda|} \zeta\left(\frac{\lambda}{|\lambda|}\right)$. Letting $\zeta_{0}=\zeta(1)$ and $\zeta_{1}=\zeta(-1)$, we get $\zeta(\lambda)=\sqrt{\lambda} \zeta_{0}$ for $\lambda>0$ and $\zeta(\lambda)=\sqrt{-\lambda} \zeta_{1}$ for $\lambda<0$.

\section{On the image of very rapidly decreasing functions under the Fourier-Weyl transform}

In this section we prove Theorem 1.5 stated in the introduction. First we recall the Euclidean result and state it for the sake of convenience. Let

$$
p_{t}(x)=(4 \pi t)^{-\frac{n}{2}} e^{-\frac{1}{4 t}|x|^{2}}, \quad x \in \mathbb{R}^{n}
$$


be the heat kernel associated to the standard Laplacian on $\mathbb{R}^{n}$. The Segal-Bargmann or the heat kernel transform is the one which takes $f \in L^{2}\left(\mathbb{R}^{n}\right)$ into the entire function

$$
f * p_{t}(z)=\int_{\mathbb{R}^{n}} f(u) p_{t}(z-u) d u, \quad z \in \mathbb{C}^{n} .
$$

Then it is well known from the work of Segal and Bargmann [2] that the image is a weighted Bergman space. More precisely, the above transformation takes $L^{2}\left(\mathbb{R}^{n}\right)$ isometrically onto the space of entire functions $F$ on $\mathbb{C}^{n}$ for which

$$
\int_{\mathbb{C}^{n}}|F(z)|^{2} p_{t / 2}(y) d x d y<\infty .
$$

In particular

$$
\int_{\mathbb{C}^{n}}\left|f * p_{t}(x+i y)\right|^{2} p_{t / 2}(y) d x d y=c_{n, t} \int_{\mathbb{R}^{n}}|f(x)|^{2} d x .
$$

We rephrase this result in the following form.

Consider functions of the form $g(x)=f(x) p_{t}(x)$ where $f \in L^{2}\left(\mathbb{R}^{n}\right)$, for a fixed $t>0$. Then

$$
\widehat{g}(\xi)=\left(f p_{t}\right) \widehat{\wedge}(\xi)=\widehat{f} * \widehat{p_{t}}(\xi) .
$$

Since $\widehat{p_{t}}(\xi)=e^{-t|\xi|^{2}}=c_{n, t} p_{\frac{1}{4 t}}(\xi)$ we see that $\widehat{g}(\xi)=c_{n, t} \widehat{f} * p_{\frac{1}{4 t}}(\xi)$ and consequently $\widehat{g}$ can be extended to $\mathbb{C}^{n}$ as an entire function. Moreover,

$$
\int_{\mathbb{C}^{n}}|\widehat{g}(\xi+i \eta)|^{2} p_{\frac{1}{8 t}}(\eta) d \xi d \eta=c_{n, t} \int_{\mathbb{R}^{n}}|\widehat{f}(\xi)|^{2} d \xi .
$$

Thus we have

Theorem 4.1. Let $t>0$ be fixed. Then a function $g \in L^{2}\left(\mathbb{R}^{n}\right)$ can be factored as $g=f p_{t}, f \in L^{2}\left(\mathbb{R}^{n}\right)$ if and only if $\widehat{g}$ is entire and satisfies

$$
\int_{\mathbb{C}^{n}}|\widehat{g}(\xi+i \eta)|^{2} p_{\frac{1}{8 t}}(\eta) d \xi d \eta<\infty
$$

Theorem 1.5 stated in the introduction is the analogue of this result for functions on the Heisenberg group. We consider functions of the form $g=f *_{3} q_{s}$ so that $g^{\lambda}(z)=f^{\lambda}(z) q_{s}^{\lambda}(z)$. Note that Theorem 1.5 is a characterisation of the image of functions $g$ for which $g^{\lambda}(z)$ has a Gaussian decay under the Fourier-Weyl transform. 
Coming to the proof we make use of the fact that

$$
q_{s}^{\lambda}(z)=c_{n}\left(\frac{\lambda}{\sinh \lambda s}\right)^{n} e^{-\frac{1}{4} \lambda(\operatorname{coth} \lambda s)|z|^{2}}
$$

(see $[\mathbf{1 1}]$ for a proof). In terms of the Euclidean heat kernel we can write

$$
q_{s}^{\lambda}(z)=c_{n}(\cosh \lambda s)^{-n} p_{s_{\lambda}}(z)
$$

where $s_{\lambda}=\lambda^{-1}(\tanh \lambda s)$. Recalling the definition of the Fourier-Weyl transform we see that

$$
\begin{aligned}
\left(\left(f *_{3} q_{s}\right)^{\sim}\right. & (\lambda, x, u) \varphi, \psi) \\
& =\int_{\mathbb{R}^{2 n}} e^{-i(x \cdot \xi+u \cdot \eta)} f^{\lambda}(\xi+i \eta) q_{s}^{\lambda}(\xi+i \eta)\left(\pi_{\lambda}(\xi+i \eta) \varphi, \psi\right) d \xi d \eta
\end{aligned}
$$

for any $\varphi, \psi \in L^{2}\left(\mathbb{R}^{n}\right)$. In view of the above expression for $q_{s}^{\lambda}$ in terms of $p_{s_{\lambda}}$, we can appeal to Theorem 4.1 to conclude that $\left(\left(f *_{3}\right.\right.$ $\left.\left.q_{s}\right)^{\sim}(\lambda, x, u) \varphi, \psi\right)$ extends to $\mathbb{C}^{n} \times \mathbb{C}^{n}$ as an entire function and that

$$
\begin{aligned}
& \int_{\mathbb{C}^{2 n}}\left|\left(\left(f *_{3} q_{s}\right)^{\sim}(\lambda, z, w) \varphi, \psi\right)\right|^{2} p_{\frac{1}{8 s_{\lambda}}}(y, v) d z d w \\
& \quad=c_{n, s_{\lambda}}(\cosh s \lambda)^{-2 n} \int_{\mathbb{R}^{2 n}}\left|f^{\lambda}(\xi+i \eta)\right|^{2}\left|\left(\pi_{\lambda}(\xi+i \eta) \varphi, \psi\right)\right|^{2} d \xi d \eta,
\end{aligned}
$$

where $z=x+i y, w=u+i v$. Letting $\psi$ run through an orthonormal basis for $L^{2}\left(\mathbb{R}^{n}\right)$ we obtain

$$
\begin{aligned}
\int_{\mathbb{C}^{2 n}} \|\left(f *_{3} q_{s}\right) & (\lambda, z, w) \varphi \|_{2}^{2} p_{\frac{1}{8 s_{\lambda}}}(y, v) d z d w \\
& =c_{n, s_{\lambda}}(\cosh s \lambda)^{-2 n} \int_{\mathbb{R}^{2 n}}\left|f^{\lambda}(\xi+i \eta)\right|^{2} d \xi d \eta \int_{\mathbb{R}^{n}}|\varphi(x)|^{2} d x .
\end{aligned}
$$

A calculation shows that

$$
(\cosh s \lambda)^{2 n} p_{\frac{1}{8 s_{\lambda}}}(y, v)=c_{n} \lambda^{-n}(\sinh 2 \lambda s)^{n} e^{-\frac{2}{\lambda}(\tanh \lambda s)\left(|y|^{2}+|v|^{2}\right)}
$$


and hence by integrating with respect to $\lambda$ we get

$$
\begin{aligned}
& \int_{-\infty}^{\infty} \int_{\mathbb{C}^{2 n}}\left\|\left(f *_{3} q_{s}\right)^{\sim}(\lambda, z, w) \varphi\right\|_{2}^{2} \omega_{s, \lambda}(y, v) d z d w d \lambda \\
& =c_{n} \int_{\mathbb{H}^{n}}|f(z, t)|^{2} d z d t \int_{\mathbb{R}^{n}}|\varphi(x)|^{2} d x,
\end{aligned}
$$

where

$$
\omega_{s, \lambda}(y, v)=c_{n} \lambda^{-n}(\sinh 2 \lambda s)^{n} e^{-\frac{2}{\lambda}(\tanh \lambda s)\left(|y|^{2}+|v|^{2}\right)} .
$$

This proves the first half of the theorem.

Conversely suppose that $\widetilde{g}(\lambda, x, u)$ extends to $\mathbb{C}^{n} \times \mathbb{C}^{n}$ as an operator valued entire function which satisfies

$$
\int_{-\infty}^{\infty} \int_{\mathbb{C}^{2 n}}\|\widetilde{g}(\lambda, z, w) \varphi\|_{2}^{2} \omega_{s, \lambda}(y, v) d z d w d \lambda<\infty \text { for all } \varphi \in L^{2}\left(\mathbb{R}^{n}\right)
$$

Then $\int_{\mathbb{C}^{2 n}}\|\widetilde{g}(\lambda, z, w) \varphi\|_{2}^{2} \omega_{s, \lambda}(y, v) d z d w<\infty$ for almost every $\lambda \in \mathbb{R}$. By the definition of $\widetilde{g}(\lambda, z, w)$ we have

$$
\begin{aligned}
& \int_{\mathbb{C}^{2 n}}\|\widetilde{g}(\lambda, z, w) \varphi\|_{2}^{2} \omega_{s, \lambda}(y, v) d z d w \\
&=\int_{\mathbb{C}^{2 n}} \int_{\mathbb{R}^{n}}\left|\int_{\mathbb{R}^{2 n}} e^{-i(x \cdot \xi+u \cdot \eta)} e^{y \cdot \xi+v \cdot \eta} g^{\lambda}(\xi+i \eta)\left[\pi_{\lambda}(\xi+i \eta) \varphi\right](\zeta) d \xi d \eta\right|^{2} \\
& \times \omega_{s, \lambda}(y, v) d \zeta d z d w .
\end{aligned}
$$

Applying Plancherel theorem in the $(x, u)$-variable this becomes

$$
\begin{gathered}
\int_{\mathbb{R}^{2 n}} \int_{\mathbb{R}^{n}} \int_{\mathbb{R}^{2 n}} e^{2(y \cdot \xi+v \cdot \eta)}\left|g^{\lambda}(\xi+i \eta)\right|^{2}\left|\left[\pi_{\lambda}(\xi+i \eta) \varphi\right](\zeta)\right|^{2} \omega_{s, \lambda}(y, v) d \xi d \eta d \zeta d y d v \\
=\|\varphi\|_{2}^{2} \int_{\mathbb{R}^{2 n}} \int_{\mathbb{R}^{2 n}} e^{2(y \cdot \xi+v \cdot \eta)}\left|g^{\lambda}(\xi+i \eta)\right|^{2} \omega_{s, \lambda}(y, v) d \xi d \eta d y d v .
\end{gathered}
$$


By the definition of $\omega_{s, \lambda}(y, v)$ the above expression becomes

$$
\begin{aligned}
&\|\varphi\|_{2}^{2} \lambda^{-n}(\sinh 2 \lambda s)^{n} \int_{\mathbb{R}^{2 n}} \int_{\mathbb{R}^{2 n}} e^{-i(y \cdot 2 i \xi+v \cdot 2 i \eta)} e^{-\frac{2}{\lambda} \tanh \lambda s\left(|y|^{2}+|v|^{2}\right)} \times\left|g^{\lambda}(\xi+i \eta)\right|^{2} d y d v d \xi d \eta \\
&=\|\varphi\|_{2}^{2} \lambda^{-n}(\sinh 2 \lambda s)^{n} \int_{\mathbb{R}^{2 n}} e^{\frac{1}{2} \lambda \operatorname{coth} \lambda s\left(|\xi|^{2}+|\eta|^{2}\right)}\left|g^{\lambda}(\xi+i \eta)\right|^{2} d \xi d \eta \\
&=\|\varphi\|_{2}^{2} \lambda^{n}(\operatorname{coth} 2 \lambda s)^{n} \int_{\mathbb{R}^{2 n}} q_{s}^{\lambda}(\xi, \eta)^{-2}\left|g^{\lambda}(\xi+i \eta)\right|^{2} d \xi d \eta<\infty
\end{aligned}
$$

i.e., $g^{\lambda}\left(q_{s}^{\lambda}\right)^{-1} \in L^{2}\left(\mathbb{C}^{n}\right)$.

Let $f_{\lambda}=g^{\lambda}\left(q_{s}^{\lambda}\right)^{-1}$ and define $f$ by the equation

$$
f(x, y, t)=\int_{\mathbb{R}} f_{\lambda}(x, y) e^{-i \lambda t} d \lambda .
$$

Then it follows that $g^{\lambda}(x, y)=f_{\lambda}(x, y) q_{s}^{\lambda}(x, y)=f^{\lambda}(x, y) q_{s}^{\lambda}(x, y)$. In otherwords, $g^{\lambda}(x, y)=\left(f *_{3} q_{s}\right)^{\lambda}(x, y)$ which means $g=f *_{3} q_{s}$ with $f \in L^{2}\left(\mathbb{H}^{n}\right)$.

\section{Acknowledgements}

This work is supported by J. C. Bose Fellowship from the Department of Science and Technology (DST). The first author is thankful to the National Board for Higher Mathematics, India, for the financial support. We would like to thank the referees for their careful reading of the manuscript and also for pointing out some inaccuracies in an earlier version.

\section{References}

[1] S. Alesker, S. Artstein-Avidan, And V. Milman, A characterization of the Fourier transform and related topics, in: "Linear and complex analysis", Amer. Math. Soc. Transl. Ser. 2 226, Amer. Math. Soc., Providence, RI, 2009, pp. 11-26.

[2] V. Bargmann, On a Hilbert space of analytic functions and an associated integral transform, Part I, Comm. Pure Appl. Math. 14(3) (1961), 187-214. DOI: 10.1002/cpa.3160140303.

[3] G. B. Folland, "Harmonic analysis in phase space", Annals of Mathematics Studies 122, Princeton University Press, Princeton, NJ, 1989. 
[4] D. Geller, Fourier analysis on the Heisenberg group. I. Schwartz space, J. Funct. Anal. 36(2) (1980), 205-254. DOI: 10.1016/0022$1236(80) 90100-7$.

[5] P. Jaming, A characterization of Fourier transforms, Colloq. Math. 118(2) (2010), 569-580. DOI: 10.4064/cm118-2-12.

[6] R. Lakshmi Lavanya and S. Thangavelu, A characterisation of the Weyl transform, Adv. Pure Appl. Math. 3(1) (2012), 113-122. DOI: $10.1515 /$ apam.2011.015.

[7] R. Lakshmi Lavanya and S. Thangavelu, A characterisation of the Fourier transform on the Heisenberg group, Ann. Funct. Anal. 3(1) (2012), 109-120.

[8] G. Mauceri, The Weyl transform and bounded operators on $L^{p}\left(\mathbb{R}^{n}\right)$, J. Funct. Anal. 39(3) (1980), 408-429. DOI: 10.1016/0022$1236(80) 90035-\mathrm{X}$.

[9] S. Thangavelu, On Paley-Wiener theorems for the Heisenberg group, J. Funct. Anal. 115(1) (1993), 24-44. DOI: 10.1006/jfan. 1993.1079.

[10] S. Thangavelu, "Harmonic analysis on the Heisenberg group", Progress in Mathematics 159, Birkhäuser Boston, Inc., Boston, MA, 1998. DOI : $10.1007 / 978-1-4612-1772-5$.

[11] S. Thangavelu, "An introduction to the uncertainty principle". Hardy's theorem on Lie groups, With a foreword by Gerald B. Folland, Progress in Mathematics 217, Birkhäuser Boston, Inc., Boston, MA, 2004. DOI : 10.1007/978-0-8176-8164-7.

R. Lakshmi Lavanya:

Ramanujan Institute for Advanced Study in Mathematics

University of Madras

Chennai-600 005

India

E-mail address: rlakshmilavanya@gmail.com

S. Thangavelu:

Department of Mathematics

Indian Institute of Science

Bangalore-560 012

India

E-mail address: veluma@math.iisc.ernet.in

Primera versió rebuda el 29 de juny de 2012, darrera versió rebuda el 15 de novembre de 2012. 